\title{
Microwave Optical Link In The Frequency Range Of 10-18 Gigahertz By Direct Modulation Of Injection Laser Diode
}

Lau, K., Yariv, A.

K. Y. Lau, A. Yariv, "Microwave Optical Link In The Frequency Range Of 10-18 Gigahertz By Direct Modulation Of Injection Laser Diode," Proc. SPIE 0545, Optical Technology for Microwave Applications II, (28 October 1985); doi: $10.1117 / 12.948338$

SPIE. Event: 1985 Technical Symposium East, 1985, Arlington, United States 
Microwave optical link in the frequency range of 10-18 gigahertz by direct modulation of injection laser diode

\author{
K.Y. Lau \\ Ortel Corporation, $2015 \mathrm{~W}$. Chestnut St., Alhambra, California 91803
}

A. Yariv

California Institute of Technology, Pasadena, California 91125

Abstract

It is demonstrated that an ultra-high speed window buried heterostructure GaAlAs laser fabricated on semi-insulating substrate can be used as narrow band signal transmitters in the Ku-band frequency range $(12-20 \mathrm{GHz})$. The modulation efficiency can be increased over a limited bandwidth by a weak optical feedback. A stronger optical feedback enables one to actively mode-lock the laser diode at a very high repetition rate up to $17.5 \mathrm{GHz}$, producing pulses of $\cong 12 \mathrm{ps}$ long.

\title{
Introduction
}

Significant progress has been made recently in operating semiconductor laser diodes at very high frequencies. A quantity of major significance in the small signal modulation regime is the -3dB modulation bandwidth, which is a direct measure of the rate at which information can be transmitted by the laser. However, one can obtain a high modulation depth at frequencies beyond the $-3 \mathrm{~dB}$ point by driving the laser with sufficient RF drive power to compensate for the drop-off in the modulation response of the laser. This technique is very useful in generating rcpetitive optical pulses from a laser diode at a very high repetition rate, although the repetition rate itself has no significance in terms of true information transmitting capacity of the laser. A means to reduce the RF drive power required for modulating the laser to a high optical modulation depth at high repetition rate is the technique of mode-locking. The laser diode is coupled to an external cavity whose round-trip time corresponds to the modulation frequency. The modulation frequency in this case is limited to a very narrow range around the round trip frequency of the external cavity. Recent experimental work have extended the small signal - $3 \mathrm{~dB}$ direct modulation bandwidth of a solitary laser diode to $\cong 12 \mathrm{GHz}$ using a specially developed window buried heterstructure laser on semi-insulating substrate (BH on SI) [1], while large signal direct modulation has been reported at repetition rates up to $10.6 \mathrm{GHz}$ [2]. Active mode-locking of laser diodes in an external cavity has been reported at repetition rates up to $7.2 \mathrm{GHz}$ in a $\mathrm{LiNbO}_{3}$ directional coupler external cavity [3] and to $10 \mathrm{GHz}$ in a fiber cavity [4].

\section{An ultra-fast laser}

The laser used in this experiment is a window BH on SI laser reported previously [1]. The length of the laser is $300 \mu \mathrm{m}$, with an active region dimension of $2 \mu \mathrm{m} x 0.2 \mu \mathrm{m}$. The presence of the window near the end facet alleviates the problem of catastrophic damage and enable the laser to operate at very high optical power densities. The tight optical and electrical confinement along the length of the laser cavity (except at the window region) enables maximum interaction between the photon and electrons to take place and results in a very high direct modulation bandwidth. The small signal modulation bandwidth of this device biased at an optical output power of $10 \mathrm{~mW}$ is approximately $10 \mathrm{GHz}$. Here, the "small signal" regime is loosely defined as that when the modulation depth of the optical output is $\lesssim 80 \%$. The $-3 \mathrm{~dB}$ bandwidth is $10.3 \mathrm{GHz}$. The response drops to $-10 \mathrm{~dB}$ at $-13.5 \mathrm{GHz}$ and to $-20 \mathrm{~dB}$ at $18 \mathrm{GHz}$ The fall-off in the modulation response is due to a combination of the intrinsic laser response and effects due to parasitic elements. However, at a limited frequency range at above the $-3 \mathrm{~dB}$ point, the response is relatively flat over a $1 \mathrm{GHz}$ band (to within $\pm 2 \mathrm{~dB}$ ), and is within $\pm 1 \mathrm{~dB}$ over a $100 \mathrm{MHz}$ bandwidth. It is thus possible to use this laser as an optical transmitter operating in a narrow bandwidth in the upper $\mathrm{x}$-band frequencies.

\section{Loss compensation and mode-locking}

The intrinsic modulation response is approximately $13 \mathrm{~dB}$ below the midband value. This loss in modulation efficincy can be partially compensated by coupling the laser to an external cavity of the appropriate length. In this experiment the external cavity is composed of a short length (6.3m) of standard graded index multimode fiber of 50um core diameter [4,5], wiht a high refractive index hemispherical lens attached to one end of the fiber to facilitate coupling. The far end of the fiber is cleaved but not metallized. The amount of opti- 
cal feedback into the laser in this arrangement is expected to be below $1 \%$, and produces no substantial reduction in lasing threshold or differential quantum efficiency. The feedback, however, induces a broad resonance in the frequency response at $\cong 16 \mathrm{GHz}$ - the round trip frequency of the fiber cavity. The full width of the resonance is about $1.5 \mathrm{GHz}$, measured at the upper anc lower $-3 \mathrm{~dB}$ points. At the peak of the resonance the modulation efficiency is enhanced by $\cong 10 \mathrm{~dB}$. The $-3 \mathrm{~dB}$ bandwidth of the resonance is approximately $1.5 \mathrm{GHz}$.

In a separate experiment the far end of a fiber is cleaved and is butted to a gold mirror. This induces a very sharp resonance in the modulation response of the laser. When the laser is driven on resonance by a microwave source whose power output is $\leqslant 6 \mathrm{dBm}$, the optical output is not fully modulated and the laser is operating in the small signal regime. As the microwave drive power is increased to > 10dBm the optical modulation depth approaches unity and the optical waveform becomes pulse-like. The characteristics of the optical pulses cannot be resolved by the photodiode, whose output appears to be sinusoidal since only the fundamental frequency $(17.5 \mathrm{GHz})$ of the modulated laser light can be detected with resonable efficiency. An autocorrelator using optical second harmonic generation is used to observe the optical output. At a microwave drive power levels of $4 \mathrm{dBm}$, the optical output is sinusoidal while at a $14 \mathrm{dBm}$ drive the output is pulse-like with a FWHM width of $12.4 \mathrm{ps}$. This, in effect, is active mode-locking of a laser diode at a repetition rate of $17.5 \mathrm{GHz}$. The spectrum of the laser consists of a large number ( $\cong 7$ ) of longitudinal modes of the laser diode since there is no frequency selective element (such as an etalon) in the external cavity. The width of the individual mode is mainly determined by frequency chirping due to heavy carrier modulation and does not seem to correspond to the transformed value of the optical pulsewidth.

\section{Conclusion}

In conclusion, we have demonstrated that suitable constructed high speed laser diodes can be used as narrow band signal transmitters in the Ku-band frequency range (12-20GHz). The modulation efficiency can be increased over limited bandwidth by a weak optical feedback A stronger optical feedback enables one to actively mode-lock the laser diode at a very high repetition rate up to $17.5 \mathrm{GHz}$, producing pulses of $\cong 12 \mathrm{ps}$ long.

This research was supported by the Defence Advance Research Project Agency, the National Science Foundation under the Optical Communication Program and by the Army Research Office.

\section{$\underline{\text { References }}$}

1. K.Y. Lau, N. Bar-Chiam, I. Ury and A. Yariv, to be published in Appl. phys. Lett., September 1984

2. C. Lin, C.A. Burrus, G. Eisenstein, R.S. Tucker, P. Besomi and R.J. Nelson, paper WJ4, Optical Fiber Communication conference, New Orleans, 1984.

3. R.C. Alferness, G. Eisenstein, S.K. Korotky, R.S. Tucker, L.L. Buhl, I.P. Kaminow

and J.J. Veselka, paper WJ3, Optical Fiber Communication conference, New Orleans, 1984.

4. R.S. Tucker, G. Eisenstein and I.P. Kaminow, Electron. Lett., 19, 552, 1983.

5. L. Figurroa, K.Y. Lau, H.W. Yen and A. Yariv, J. Appl. Phys., 51, 3062, 1980.

6. J.E. Bower and C.A.Burrus, paper M-1, Ninth Inter. Semicond. Laser Conf., Rio de Janeiro, 1984

7. R.A. Linke, paper M-4, Ninth Inter. Semicond. Laser Conf., Rio de Janeiro, 1984. 